College, Oxford, to prepare for a study of the growth of voluntary organizations providing a structural basis for democratic society in French and/or British West Africa.

\title{
Institute of African-American Relations
}

THIs Institute, with headquarters in Washington, D.C., has as its aims the strengthening of African-American relations, and in particular the assisting of African students in the United States. To this end it has since January 1955 operated a Scholarship Program whereby financial assistance is provided for African students resident at an educational institution in the United States. It has also organized an essay competition, offering prizes for essays by African students on the subject of African-American Relations. Six prizes were awarded, while seven essays received honourable mention. The winner of the first prize, a Nigerian, is studying for the degree of M.A. in Education at the University of Chicago. The Institute plans to open an African House in Washington, to serve as a place of meeting, study, and recreation for African students as well as a centre for programmes for local Americans. The Institute publishes a periodical Bulletin.

\section{L'Éducation de Base}

M. Le Gouverneur Deschamps a présenté une communication au sujet de l'éducation de base devant le Comité Central de la France d'Outre-mer, le 25 mars 1959 . L'éducation de base, dit M. Deschamps, est une méthode d'éducation totale des collectivités arriérées. Il s'agit d'apprendre à ces collectivités, non seulement les rudiments de l'écriture, de la lecture et du calcul, mais les méthodes permettant d'améliorer leur sort en général, c'est à dire, l'hygiène, la construction, l'agriculture, l'élevage, tout ce qui touche à leur mode de vie traditionnel. Ce système avait pris naissance dans le Mexique et s'était passé à UNESCO, qui avait essayé d'étendre ce plan à d'autres pays sous-développés. Des centres internationaux avaient été créés par l'UNESCO en Amérique et dans le Moyen-Orient; l'efficacité de cette action internationale était encore discutable, mais elle avait éveillé l'idée de l'éducation de base, et la Commission Culturelle de l'UNESCO avait créé un sous-comité présidé par M. Senghor et le Professeur Rivet. Ce sous-comité avait permis d'apprendre un certain nombre de choses sur la manière dont les expériences se déroulaient de par le monde, ainsi que l'intérêt qu'elles présentent. Dans les territoires français de l'Afrique un premier essai avait été fait à M'Boumba en I95 I; un autre avait eu lieu à Darau Mousti (voir Africa, xxv, 3, 1955, p. 289), région où les gens étaient nettement hostiles à l'instruction. Néanmoins, l'équipe d'Éducation de Base avait réussi à créer des contacts: des champs avaient été mis en culture, le bétail avait été vacciné, les malades avaient été soignés. D'autres expériences étaient en cours au Sénégal, en Côte d'Ivoire, au Dahomey, au Togo. Un centre de moniteurs d'Éducation de Base a été créé en A.O.F., en vue de les préparer à ces techniques tout à fait particulières. Au Cameroun avait été créé un Comité Territorial et un Bureau de l'Éducation de Base, dépendant de la Direction de l'Enseignement, sous la direction de M. Pauvert, sociologue, qui avait procédé à des enquêtes sociales préliminaires très étendues. Une formule originale était le Journal de Nyong et Sanaga, lancé par M. Pauvert-un journal de villages édité par les gens du pays (voir Africa, xxiv, 1, 1954, p. 63). En A.E.F. il y avait eu la tentative de M. Fourré (voir Africa, xxv, 1, 1955, p. 95) mais, sauf au Sénégal et au Cameroun, il n'existait pas d'organisation.

La création du Conseil Supérieur de l'Éducation de Base, et l'affectation d'une somme de 800 millions destinée à une période de quatre ans marquaient une étape dans l'histoire de l'Éducation de Base dans les territoires français.

Le Conseil Supérieur de l'Éducation de Base a tenté de dégager une doctrine qui comprend 